the description of flow through porous materials - 'the percolation problem'.

The experiment of Nittmann et al. is also significant in that it gives insight into the minimum requirements of a DLA-like structure. Although DLA seems quite simple, it has defied complete analysis. A major point of contention is whether noise due to the discrete nature of the particles making up the cluster dominates the dynamics. Dielectric breakdown structures have links of more-or-less fixed length and electrolytic deposits are polycrystalline. Experiments on these systems shed no light on the question. But the fluid system of Nittman et al. is continuous. Apparently, discreteness is not essential to make a DLAlike fractal. There has been speculation that this would indicate that DLA is an example of chaos. In practical terms it may mean that solidification far from equilibrium, which can also be mapped onto DLA, could give rise to fractals - for example, if a snowflake were allowed to grow very large, it would probably be fractal, too.

1. Nittmann, J., Daccord, G \& Stanley, H. E. Nature 314, 141 (1985).

Brady, R.M. \& Ball, R.C. Nature 309, 225 (1984)

3atsushita. M., Sano, M., Hayakawa, Y., Honjo, H. \& Sawada, Y. Phys. Rev. Lett. 53, 286 (1984)

4. Niemeyer, L., Pietronero, L. \& Wiesmann, H. J. Phys. Rev. Lett. 52, 1033 (1984).

5. Mandelbrot, B. The Fractal Geometry of Narure (Freeman San Francisco, 1982).

6. Saffman, P.G. \& Taylor, G.1. Proc. R. Soc. A245, 312 (1958).

7. Paterson, L. Phys. Rev. Lett. 52, 1621 (1984).

8. Witten, T.A. \&ander, L.M. Phys. Rev. Lett. 47, 1499 (1981); Phys. Rev. B27, 5685 (1983)

L.M. Sander is in the Physics Department, University of Michigan, Ann Arbor, Michigan 48109-1120. USA.

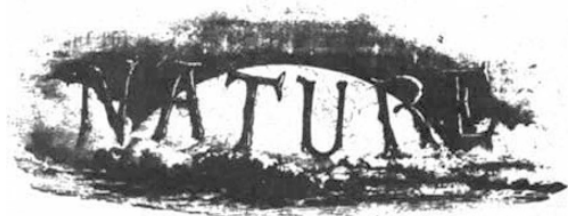

100 Years Ago

THE following account, we learn from Science, of unusual phenomena was received, March 10, at the Hydrographic Office, Washington, from the branch office in San Francisco. The barque Innerwich, Capt. Waters, has just arrived at Victoria from Yokohama. At midnight of February 24 , in latitude $37^{\circ}$ north, longitude $170^{\circ} 15^{\prime}$ east, the captain was aroused by the mate, and went on deck to find the sky changing to a fiery red. All at once a large mass of fire appeared over the vessel, completely blinding the spectators; and, as it fell into the sea some fifty yards to leeward, it caused a hissing sound, which was heard above the blast, and made the vessel quiver from stem to stern. Hardly had this disappeared when a lowering mass of white foam was seen rapidly approaching the vessel. The barque was struck flat aback; but, before there was time to touch a brace, the sails had filled again, and the roaring white sea had passed ahead. The master declares that the awfulness of the sight was beyond description and considers that the ship had a narrow escape from destruction.

From Nature 31 514, 2 April 1885.

Oceanography

\title{
Sonar classification of sea beds
}

\author{
from M.L. Somers
}

Although side-scan solar is used widely in both scientific and engineering investigations of the sea bed, the task of extracting a numerical classification of sea beds from the backscattered sound has proved intractable. But an experienced human observer who has had access to the results of comparisons between sonographs and physical samples can rapidly make accurate qualitative classifications, so there must be some quality of the backscattering that gives the necessary clues. On page 426 of this issue, Z. Reut, N.G. Pace and M.J.P. Heaton offer a solution to the problem and a method of classification into six sea-bed types - mud, sand, clay, gravel, stones and boulders.

The backscattering of sound from the sea bed is a complicated function of the grazing angle of the sonar signal and the roughness of the sea bed, with further complications thrown in by the acoustic impedance of the sea-bed material: if the ratio of sea bed to water impedance is close to unity (as in the case of mud), then there is appreciable penetration of sound into the sea bed and the possible production of a boundary wave, which can interact with the incident wave, profoundly modifying the backscattering process (see Somers, M.L. and Stubbs, A.R. IEE Proc. 131, Pt F; 1984). To circumvent these problems, Reut et al. have concentrated on the rate of fluctuation of the backscattered sound over short periods of time. Their method is based on the observation that the fluctuations generally become more rapid as the ground gets rougher. There are, of course, large differences in total energy, and the peakto-valley ratios can actually be greater over a soft or smooth sea bed than over a rough one, but the rate of fluctuation is still generally less than if the sea bed is rough.

The method requires fairly careful normalization, for which Reut et al. have chosen to use cepstrum analysis, an outline of which is given in their paper. By taking the mean of the power spectrum, all considerations of phase can be ignored, so ensuring that when the inverse transform is taken the result peaks at, or near to, zero time delay. The effect of taking logs before doing the inverse transform is to deemphasize any d.c. level in the signal, which shows up as a large peak at zero frequency in the power spectrum. The twopower cepstrum integral parameters give a measure of how closely the cepstrum is confined to low time-lag values.

Justification of the method comes both from the foregoing qualitative arguments that can be mustered in its favour and from the fact that it seems to work - 120 seabed areas are correctly classified into the six types with the only overlap being bet- ween gravel and stone types. A similar result could probably be obtained by applying automatic gain control, removing the mean and taking the auto-correlation function, though this would possibly be computationally less attractive.

It would be interesting to see the method applied to a wider selection of sea-bed echoes, using a number of frequencies instead of the single $48-\mathrm{kHz}$ frequency used by the authors. An application which comes to mind concerns the problem of mapping and assessing fields of manganese nodules in oceans that are several thousand metres deep. This will require lowfrequency sound, such as used in the longrange side-scan sonar, GLORIA II. Manganese nodule fields occur very widely in the Abyssal Plain regions of the deep ocean and should be visible on a GLORIA record. If so, it might at least be possible to delineate the boundaries of the fields, and perhaps even to give a measure of nodule abundance.

Side-scan sonar requires oblique incidence for its best visual effect, so that depth/range ratios need to be less than about 0.2 . But most systems working from the surface in deep water, or at high frequencies in shallow water, operate at nearvertical incidence and the backscattering process is somewhat different. It will be interesting to see how the classification system of Reut et al. performs in such circumstances.

Turning from their concern with problems of propagation and surface reverberation, the underwater-acoustics community is devoting increasing attention to sonar scattering. Many theoretical papers and some reporting measurements or equipment will be given at the International Conference on Scattering Phenomena on 2-3 April at the Admiralty Research Establishment, Portland, UK. Various applications of the analysis of sea-bed backscattering, both civil and military, are being explored. Of the civil applications, many involve the estimation of resources. Manganese nodules have already been mentioned. Gravel provides a second example. Over ten per cent of the gravel used in the United Kingdom is dredged from the sea bed, and a rapid and reliable means of detecting suitable gravel fields would be of considerable value. The accuracy of acoustic logs for ships that measure their speed by observing the Doppler shift of sound backscattered from the sea bed should also be improved by application of the new system of classification.

M.L. Somers is at the Institute of Oceanographic Sciences, Wormley, Godalming, Surrey GU8 $5 \cup B, U K$. 